\title{
Challenges of deprescribing in the multimorbid patient
}

\author{
Shane Cullinan, ${ }^{1}$ Christina Raae Hansen, ${ }^{1}$ Stephen Byrne, ${ }^{1}$ Denis O'Mahony, ${ }^{2}$ \\ Patricia Kearney, ${ }^{3}$ Laura Sahm ${ }^{1}$
}

${ }^{1}$ School of Pharmacy, University College Cork, Cork, Ireland

${ }^{2}$ Department of Geriatric Medicine, Cork University Hospital, Cork, Ireland

${ }^{3}$ Department of Epidemiology and Public Health, University College Cork, Cork, Ireland

\section{Correspondence to} Dr Shane Cullinan, School of Pharmacy, University College Cork, Cork, Ireland;

Shane.cullinan@ucc.ie

Received 15 February 2016

Revised 25 April 2016

Accepted 28 April 2016
CrossMark

To cite: Cullinan S, Raae
Hansen C, Byrne S, et al.
Eur J Hosp Pharm
2017;24:43-46.

\begin{abstract}
Older patients often have multimorbidity, frequently resulting in polypharmacy. Independently, multimorbidity and polypharmacy are among the biggest risk factors for inappropriate medication, adverse drug reactions, adverse drug events and morbidity, leading to patient harm and hospitalisations. After a medication review, discontinuation of medication or deprescribing is one of the most common recommendations but is likely to be ignored. The deprescribing process includes some or all of the following elements: a review of current medications, identification of medications to be discontinued, a discontinuation regimen, involvement of patients and a review with follow-up. In addition to the complexity presented by prescribing or deprescribing for older multimorbid patients, other factors act as barriers to discontinuation of medications in these patients; these include interprofessional relationships, difficulties with medication reviews, deficiencies in knowledge and evidence and patients' preferences/resistance to change. These challenges are compounded by the need to manage the shared treatment of multiple conditions by several prescribers from different specialties based on disease-specific guidelines without evidence of effects on the older, frailer, multimorbid patients. The interdisciplinary effort in the treatment of such patients needs to improve to ensure that we treat the patient holistically and not just the individual conditions of the multimorbid patient, according to guidelines. We must first, however, equip prescribers to identify instances where deprescribing is appropriate and then make the necessary changes to pharmacotherapy.
\end{abstract}

\section{INTRODUCTION}

Within the 34 member countries of the Organisation for Economic Co-operation and Development (OECD), people born today have an average life expectancy of 80.1 years. ${ }^{1}$ This is a 10 -year increase compared with just 45 years ago. Sixty-five year olds today have an average life expectancy of 19.25 years, almost a 6-year increase from 1960. Of these extra 19 years, nine are likely to be 'healthy years'. ${ }^{2}$ In $1960,8.6 \%$ of the OECD population was aged $\geq 65$ years. Today, that figure is $15.4 \%$ and set to rise to $27.2 \%$ by $2050 .^{3}{ }^{4}$ The global population is ageing. With this come many socioeconomic burdens and increased pressures at all levels of care.

Older patients often have to contend with multimorbidity, which in turn leads to polypharmacy. Together, multimorbidity and polypharmacy are among the biggest risk factors for inappropriate medication, adverse drug reactions (ADRs), adverse drug events and morbidity, leading to patient harm and hospitalisations. ${ }^{5}{ }^{6}$ Suboptimal prescribing in older patients has been well-established as a significant problem in healthcare today. ${ }^{7-9}$ In recent years, the focus of research into optimisation of medicines for older patients has shifted from quantitatively measuring the deficiencies in prescribing for this cohort, to qualitatively uncovering the root causes of suboptimal prescribing.

Instead of asking how bad the problem is, attention is now turning to why does it happen and how can we deal with it? Published reports of qualitative research attempting to answer and deal with these questions have increased. ${ }^{10-13}$ From this research, new avenues for exploration have emerged that may optimise prescribing for older multimorbid patients through targeted interventions and new procedures for medication reviews. ${ }^{14-16}$ However, one of the most common recommendations after a medication review-discontinuation of medication or deprescribing - is one of the least likely to be followed. ${ }^{17}{ }^{18}$ The deprescribing process includes some or all of the following elements: a review of current medications, identification of medications to be discontinued, a discontinuation regimen, involvement of patients and a review with follow-up. ${ }^{19}$ Our review highlights some of the potential reasons for this lack of deprescribing and the challenges to discontinuing drugs for these patients.

\section{WHY DEPRESCRIBE?}

Recent research by our group examined the effect of a structured pharmacist review on the appropriateness of medications as well as adverse outcomes such as ADRs in patients with multimorbidity and polypharmacy. ${ }^{15}{ }^{16}$ In a cluster randomised controlled trial, patients in the intervention group underwent a thorough medication review by a pharmacist using a computerised decision support system (CDSS) to aid the generation of recommendations. Of the 577 recommendations made in 296 patients about the appropriateness of pharmacotherapy, 297 (51\%) advised stopping at least one medication, based on the Screening Tool of Older Person's Prescriptions (STOPP) criteria. $^{20} 21$ The results of the recommendations were (i) an improvement in overall appropriateness of medications (illustrated by a significant improvement in Medication Appropriateness Index score), (ii) a significant improvement in ADR rates in the intervention group compared with the control group (13.9\% vs $20.7 \%, p=0.02)$, (iii) a shorter hospital stay but with no statistically significant difference between the groups ( 8 vs 9 days, $\mathrm{p}=0.44$ ). While discontinuation of medications was not the only 
'appropriateness' recommendation provided to medical teams, it was the most common and the most widely implemented. However, acceptance rates were still only $45 \%$. Fewer than half the deprescribing recommendations were implemented, yet significant improvements in ADR rates and medication appropriateness were still achieved. Improving the acceptance rates would therefore appear to be a justified exercise.

\section{CHALLENGES TO DEPRESCRIBING}

The low acceptance rates of deprescribing recommendations mentioned above and elsewhere, ${ }^{17} 18$ invite inquiry into the reasons why despite the potential benefits. Deprescribing is a difficult task for practitioners in all patients but is further complicated in older multimorbid people owing to the need to consider life expectancy in addition to age-related changes in pharmacokinetics (PK) and pharmacodynamics (PD). ${ }^{22} \mathrm{PK} / \mathrm{PD}$ changes are important indicators of deprescribing, enabling us to distinguish between drug-related adverse events and general age-related symptoms and identification of drugs and doses that are potentially inappropriate. Prescribing and deprescribing should be regarded as equally important in considering the drug-induced harm that is to be ameliorated or prevented through deprescribing, the benefits and risks and the assessment and management of the withdrawal. ${ }^{23}$ The same insight and understanding of a patient's clinical situation is thus required to facilitate both safe prescribing and deprescribing.

Despite existing tools such as STOPP, Beer's Criteria, Medication Appropriateness Index and Medstopper and clinical guidelines on safe withdrawal of drug dependence to guide discontinuation of inappropriate drugs safely, there is still a gap in the management of polypharmacy and the use of drugs for chronic conditions where therapeutic alternatives do not exist. ${ }^{24}$

However, factors other than complexity play a part, which contribute to prescribers' reluctance to deprescribe.

\section{INTERPROFESSIONAL RELATIONSHIPS}

In the treatment of multimorbid older patients, the involvement of several healthcare professionals is common. ${ }^{25-32}$ This often results in individuals following his/her specialty treatment guideline(s) and dominating the patient's treatment with their own particular focus. ${ }^{26}$ Similarly, some physicians believe that they are solely responsible for the medicines management within their specialty and that the overall management is the responsibility of others. ${ }^{31}$ Lack of communication between the various levels of care is a known source of suboptimal prescribing. ${ }^{11} 12$

The literature abounds with studies illustrating this confusion over where responsibility lies. In some instances, it has been shown that primary care physicians welcome the help of pharmacists to support them in polypharmacy management and most pharmacists are in favour of the suggested clinical role for them in treating multimorbidity. ${ }^{31}$ However, in other instances the perceived value of a pharmacist's recommendations varies between the general practitioners (GPs), ${ }^{32}$ and seems to be determined by the relationship between the medical and the pharmacy profession. In one study it was shown that junior doctors felt that GPs and consultants were the main healthcare professionals responsible for deprescribing, followed by senior house officers, junior doctors and pharmacists. ${ }^{33}$ Recent literature reviews of pharmacy-led interventions ${ }^{34}$ have described a positive impact on the appropriateness of prescribing in older patients. Promising results were reported from both interventions of pharmacists working independently or as part of a multidisciplinary team. Despite different levels of clinical significance of the interventions reviewed, both reviews highlighted the important role of pharmacists in improving the quality of medication use among older patients.

Elsewhere, it has been reported that GPs feel that the responsibility to review a patient's overall health status and quality of life is theirs. Hence, they believe that they have a coordinating role in reviewing the patient's medical treatment, including lowering the doses, quantifying the medication use and reducing the number of inappropriate drugs. However, they also described the challenges of these tasks, which include a heavy workload on top of their regular work.

It's a great idea to reduce medication if you can do it in a safe manner that's not going to make us have to go out to the nursing homes 55 more times: $:^{32}$

Another factor is a reluctance to interfere with medication that has been prescribed by a colleague or a specialist. ${ }^{32}$ Our research group has encountered this, both through our work in developing and implementing the STOPP/Screening Tool to Alert doctors to Right Treatment (START) criteria $^{20} 21$ and our qualitative work exploring the barriers to appropriate prescribing in older patients. ${ }^{10}$ Prescribers described a fear of offending other doctors, including specialist doctors and GPs. ${ }^{10}$ If, for example, a doctor noticed something potentially inappropriate in a patient's prescription, they would be less likely to intervene if that patient was under the care of a specialist. Similarly, when transferring information -for example, from hospital to primary care, it was noted that information might be limited owing to a fear of causing offence to patients' GPs. This fear of offending other doctors is compounded by a fear of upsetting a medication regimen. There is a culture of 'don't rock the boat' when it comes to making changes.

Doctors described reluctance to discontinue a medication that has been taken for a long time by a patient in order to avoid worry and spoke of not wishing to disrupt patients' clinical stability. ${ }^{10}$

It can be argued that the medical treatment of chronic diseases takes place after hospital discharge and that problems of polypharmacy and inappropriate medication use are resolved in primary care. The GPs may therefore be the key players. However, the focus of the current deprescribing debate is on hospital specialists who are called to "take the lead in deprescribing". ${ }^{23}$ The reported reluctance among practitioners to interfere with decisions made by a specialist highlights a need to change the medical culture and improve the communication between levels of care. The transition between primary and secondary care is often associated with miscommunication and a lack of clarity of the roles and responsibility for a patient's medical treatment, including deprescribing. A debate as to who needs to take the lead in deprescribing and in what setting the process should take place may therefore involve a discussion on improving collaboration between levels of care to optimise safe medication use. This cultural change and discussion applies to the physicians and practitioners, but might also be relevant to the roles of hospital pharmacists and pharmacists in primary care.

\section{MEDICATION REVIEW DIFFICULTIES}

Before any deprescribing takes place, a thorough medication review needs to be carried out, which is often challenging in the older multimorbid patient. Multiple prescribers usually mean that a clear overview of the patient's medical treatment is difficult to achieve. $^{26} 2931$ This is further compounded by the lack of interprofessional communication described above, in 
particular poor documentation of changes made to a treatment - for example, initiation, amendments and discontinuation. In turn, this poor documentation is a barrier to deprescribing as it hinders the understanding of other doctors' motivations for the initiation or continuation of a particular treatment. ${ }^{26} 293132$

Apart from poor documentation by prescribers, the difficulty in obtaining an updated list of patients' medications further hinders deprescribing. Shemeili et $a l^{31}$ reported that the main difficulty with medication review was the need to consult several sources-for example, pharmacy, patient, family and GP, to complete the list, coupled with uncertainty about which source provides the optimal list of drugs taken by the patient. Not knowing which medications should be included on the list -for example, 'as required' (PRN) analgesics or topical medicines, was another challenge mentioned. ${ }^{31}$ The literature also describes how incomplete information is perceived as a barrier to making a decision about deprescribing - that is, which drugs to discontinue and when. ${ }^{30}$ Poor acquisition and documentation of patient information from nurses has also been suggested as a barrier to deprescribing:

It's a matter of educating them [nursing staff] that they need to provide information about that resident that's documented well and correctly so that we can use that information. ${ }^{32}$

A medication review is a critical step in assessing a patient's pharmacotherapy and ultimately deprescribing for that patient, and warrants attention. Our group has investigated the use of a CDSS-supported, Structured Pharmacist Review of Medication (SPRM) and the Structured HIstory taking of Medication use (SHIM) tool to streamline the medication review process. Both approaches improved the accuracy and reliability of patient data obtained. ${ }^{15} 1635$ We did find, however, that any pro forma used, still relied on basic communication between levels of care and adequate documentation by prescribers.

\section{KNOWLEDGE AND EVIDENCE BASE}

Simply not knowing what can be safely discontinued or indeed what should be discontinued is in itself a barrier to deprescribing. We have found that medical students and junior doctors are not equipped to make these decisions about older patients as there is little or no distinction made between them and the general adult population in their training, ${ }^{12}$ even though they are entirely different populations. As previously mentioned, altered PKs and PDs, reducing renal and hepatic function and altered body fat/lean muscle ratios all make prescribing for older patients notoriously difficult. If physicians are not being trained to prescribe for them, how can we expect them to effectively deprescribe for them?

\section{It's a different knowledge set. And it's difficult you know because there isn't a huge amount of data out there or its not communi- cated to us very well ${ }^{12}$}

The lack of evidence for the use of or discontinuation of a particular drug by older patients limits structured deprescribing, mainly owing to the exclusion of multimorbid older patients in clinical trials. ${ }^{25-27} 30$ The available evidence is perceived by many as insufficient in relation to the effect of multiple drug treatments in older patients ${ }^{25}$ and the effects of preventive medication in the oldest patients. ${ }^{26} 27{ }^{30}$ A low level of evidence often underlies recommendations in existing treatment guidelines and many commonly used recommendations are based on expert opinions and 'standard of care'. ${ }^{36} 37$ As a result, although there is an abundance of prescribing guidelines, their application to older, multimorbid patients is unsatisfactory for the following reasons:

1. They are based on trial data involving younger patients. ${ }^{30}$

2. They provide only a standardised set of recommended medications for each indication regardless of a patient's additional comorbidities. $^{30}$

3. They are too disease-specific. ${ }^{38}$

4. They do not include recommendations for deprescribing. ${ }^{27}$

Despite these misgivings, in the absence of alternative evidence, many clinical guidelines become widely used and prescribers feel under pressure to adhere to them instead of prioritising the medical treatment and deprescribing where appropriate. $^{262930}$ A prescriber's tendency to deprescribe may therefore be affected by the lack of evidence-based guidelines and also by the particular medical culture. The lack of guidance for the multimorbid patient warrants further research. Until evidence-based recommendations are incorporated into succinct and validated guidelines, any efforts to systematically deprescribe will be based on the same 'expert opinion' approach previously seen. Although this has its benefits, a more standardised and robust system for optimising a patient's prescriptions is required. A barrier to this has been the exclusion of older, multimorbid patients from clinical trials but, encouragingly, two ongoing trials are focusing on these very patients. The SENATOR trial is assessing the impact of a CDSS, incorporating the STOPP/START criteria, on ADR rates in older multimorbid patients (http://www.senator-project.eu/). The OPERAM project is also assessing the impact of a CDSS in these patients, with drug-related admissions as the primary outcome. It is hoped that the findings from these trials will facilitate a significant step towards evidence-based prescribing guidance for multimorbid patients. Evidence, or lack of evidence, of ADRs is also something that influences prescribing decisions. Damestoy et al ${ }^{39}$ reported that many of the physicians interviewed prescribed as they did because they did not often see side effects. Dickinson et $a l^{40}$ reported that GPs did not perceive a significant problem with long-term prescribing of antidepressants as they had not seen any evidence to indicate serious harm to the older patient. What is not clear in these instances is whether ADRs simply were not occurring or the prescriber was just not aware of them.

\section{PATIENT}

The patient has an important influence on the deprescribing process because:

1. Some patients' unintentionally withhold information about adverse drug events because they attribute these to ageing rather than the side effects of medicines. ${ }^{26}$

2. Some patients are more likely to report their symptoms to healthcare professionals such as hospital specialists or nurses other than their GPs, which means that the GP is not being fully aware of the problems experienced by the patient ${ }^{26}$

3. Patient characteristics, such as cognitive impairment, functional dependency, level of education and old age, hinder a patient's explanation of problems with their current medications and the need for deprescribing. ${ }^{25} 26$

4. Some patients do not wish to stop familiar medications. ${ }^{26} 29$

5. Patients' demands, wishes and expectations and those of their families may have an influence. 2529303238

6. Practitioners are reluctant to talk to patients about their life expectancy. $^{26}$

In this research group's experience, all of the above can be significant obstacles to deprescribing-particularly, points (4) and (5). We have found that doctors are commonly influenced 
by patients or patients' families when it comes to prescribing, which often results in them prescribing something that they know may not be technically appropriate or even required. ${ }^{10} 12$

Many of the physicians thought patients would seek out another physician if they were not satisfied with their prescription and they took this into account before prescribing. ${ }^{10}$

As a doctor sometimes, you feel that you have to do something, you get pressurized by either nursing staff, relatives or patients. You have to give them something. So you end up giving something that you are not $100 \%$ happy with. ${ }^{12}$

These outside influences are difficult to deal with. However, procedures for teaching medical students 'non-technical skills', such as dealing with patients and their families, have recently been proposed, ${ }^{41}$ and would appear to be warranted. There is also a consensus among the medical profession that increased targeted patient education would help to improve communication between doctor and patient. ${ }^{12}$

\section{OVERCOMING THE CHALLENGES}

Dealing with the challenges outlined above by improving the lines of communication between levels of care, making it explicit who has the ultimate responsibility for ensuring the appropriateness of a patient's medical treatment, improving the medication review processes, including the patient in the decision-making process, better educating our young doctors and patient education, will all aid deprescribing.

However, two areas should be the focus of immediate attention and the hospital pharmacist is ideally suited to deal with both. In order to facilitate deprescribing, we must (i) be able to identify instances where discontinuation of medications would be appropriate and (ii) know what to change and how. Several sets of explicit criteria have been developed to aid in the identification of instances where deprescribing would be beneficial. The two most established are the Beers criteria, ${ }^{42}$ mainly used in the USA, and the STOPP/START criteria, ${ }^{20} 21$ developed by our team in Cork. While these are useful tools and describe clear, practice-based situations where deprescribing might be beneficial, we have found that doctors are either unaware of them or do not know how to implement them. ${ }^{12}$ Providing prescribers with information about these tools and training is critical. To know what to do once polypharmacy/inappropriate prescribing is detected and to know what to discontinue requires experience. But doctors could be given a much better start than is currently the case. Major deficiencies in geriatric pharmacotherapy training have been uncovered through interviews with doctors. ${ }^{12}$ Prescribers need to be made aware at an earlier stage the vast differences between older and younger patients.

\section{CONCLUSION}

The challenges of deprescribing in older patients are compounded by the need to manage the shared treatment of multiple conditions by several prescribers from different specialties based on disease-specific guidelines which do not contain evidence on the older, frailer, multimorbid patient population. Interdisciplinary effort in the treatment of older patients with multimorbidity needs to be improved to make sure that we treat the patient holistically and do not merely treat the individual conditions. We must first, however, equip prescribers to identify instances where deprescribing is appropriate and then make the necessary changes to pharmacotherapy.
Contributors $\mathrm{CRH}$ : wrote the paper, researched the topic, consulted experts in the field. SB, DO, PK, LS: contributed to research, contributed own findings and experiences, aided in writing of paper. SC: co-wrote the paper, reviewed the final draft, researched the topic, contributed to paper design and style.

Funding Swiss State Secretariat for Education, Research and Innovation (SERI) (grant number 15.0137); the European Commission (EC) HORIZON 2020 (grant number 634238).

Competing interests None declared.

Provenance and peer review Not commissioned; externally peer reviewed.

\section{REFERENCES}

1 OECD. Life expectancy at birth. 2015. OECD Publishing.

2 OECD. Life expectancy and healthy life expectancy at age 65. 2015. OECD Publishing.

3 OECD. Share of the population aged 65 and over, 1960 to 2011. 2011. OECD Publishing.

4 OECD. Demographic trends. 2016. OECD Publishing.

5 Olsson IN, Runnamo R, Engfeldt P. Medication quality and quality of life in the elderly, a cohort study. Health Qual Life Outcomes 2011;9:95.

6 Centers for Medicare and Medicaid Services. Chronic Conditions Among Medicare Beneficaries [MD]. Baltimore, 2012. Edition 2012. file:///C:/Users/Christina/Dropbox/ PhD20Uddannelse/Literature20Review_Deprescribing/Introduction_discussio_ literature/2012Chartbook.pdf

7 Gallagher P, Barry P, O'Mahony D. Inappropriate prescribing in the elderly. J Clin Pharm Ther 2007;32:113-21.

8 Gallagher P, Lang PO, Cherubini A, et al. Prevalence of potentially inappropriate prescribing in an acutely ill population of older patients admitted to six European hospitals. Eur J Clin Pharmacol 2011;67:1175-88.

9 O'Mahony D, Gallagher PF. Inappropriate prescribing in the older population: need for new criteria. Age Ageing 2008;37:138-41.

10 Cullinan S, O'Mahony D, Fleming A, et al. A meta-synthesis of potentially inappropriate prescribing in older patients. Drugs Aging 2014;31:631-8.

11 Spinewine A, Swine C, Dhillon S, et al. Appropriateness of use of medicines in elderly inpatients: qualitative study. BMJ 2005;331:935.

12 Cullinan S, Fleming A, O'Mahony D, et al. Doctors' perspectives on the barriers to appropriate prescribing in older hospitalized patients: a qualitative study. $\mathrm{Br} J \mathrm{Clin}$ Pharmacol 2015;79:860-9.

13 Clyne B, Bradley MC, Hughes CM, et al. Addressing potentially inappropriate prescribing in older patients: development and pilot study of an intervention in primary care (the OPTI-SCRIPT study). BMC Health Serv Res 2013;13:307.

14 Cullinan S, O'Mahony D, O'Sullivan D, et al. Use of a frailty index to identify potentially inappropriate prescribing and adverse drug reaction risks in older patients. Age Ageing 2016;45:115-20.

15 O'Sullivan D, O'Mahony D, O'Connor MN, et al. The impact of a structured pharmacist intervention on the appropriateness of prescribing in older hospitalized patients. Drugs Aging 2014;31:471-81.

16 O'Sullivan D, O'Mahony D, O'Connor MN, et al. Prevention of adverse drug reactions in hospitalised older patients using a software-supported structured pharmacist intervention: a cluster randomised controlled trial. Drugs Aging 2016;33:63-73.

17 Kroenke K, Pinholt EM. Reducing polypharmacy in the elderly. A controlled trial of physician feedback. J Am Geriatr Soc 1990;38:31-6.

18 Brulhart MI, Wermeille JP. Multidisciplinary medication review: evaluation of a pharmaceutical care model for nursing homes. Int I Clin Pharm 2011;33:549-57.

19 Woodward M. Deprescribing: achieving better health outcomes for older people through reducing medictions. J Pharm Pract Res 2003;33:323-8.

20 Gallagher P, Ryan C, Byrne S, et al. STOPP (Screening Tool of Older Person's Prescriptions) and START (Screening Tool to Alert doctors to Right Treatment). Consensus validation. Int J Clin Pharmacol Ther 2008;46:72-83.

21 O'Mahony D, O'Sullivan D, Byrne S, et al. STOPP/START criteria for potentially inappropriate prescribing in older people: version 2. Age Ageing 2015;44:213-18.

22 Hardy JE, Hilmer SN. Deprescribing in the last year of life. J Pharm Pract Res 2011;41:146-51

23 Scott IA, Le Couteur DG. Physicians need to take the lead in deprescribing. Intern Med J 2015;45:352-6.

24 Budnitz DS, Lovegrove MC, Shehab N, et al. Emergency hospitalizations for adverse drug events in older Americans. N Engl J Med 2011;365:2002-12.

25 Ni Chróinín D, Ni Chróinín C, Beveridge A. Factors influencing deprescribing habits among geriatricians. Age Ageing 2015:44:704-8.

26 Schuling J, Gebben $H$, Veehof $L$, et al. Deprescribing medication in very elderly patients with multimorbidity: the view of Dutch GPs. A qualitative study. BMC Fam Pract 2012;13:56.

27 Farrell B, Tsang C, Raman-Wilms L, et al. What are priorities for deprescribing for elderly patients? Capturing the voice of practitioners: a modified Delphi process. PLOS ONE 2015;10:e0122246. 
28 Harriman K, Howard L, McCracken R. Deprescribing medication for frail elderly patients in nursing homes: a survey of Vancouver family physicians. BC Med J 2014;56:436-41.

29 Anthierens S, Tansens A, Petrovic M, et al. Qualitative insights into general practitioners views on polypharmacy. BMC Fam Pract 2010;11:65-70.

30 Moen J, Norrgård S, Antonov K, et al. GPs' perceptions of multiple-medicine use in older patients. J Eval Clin Pract 2010;16:69-75.

31 Shemeili SA, Klein S, Strath A, et al. An exploration of health professionals' experiences of medicines management in elderly, hospitalised patients in Abu Dhabi. Int J Clin Pharm 2015;1:107-18.

32 Palagyi A, Keay L, Harper J, et al. Barricades and brickwalls - a qualitative study exploring perceptions of medication use and deprescribing in long-term care. BMC Geriatr 2016;16:15

33 Jubraj B, Marvin V, Poots AJ, et al. A pilot survey of junior doctors' attitudes and awareness around medication review: time to change our educational approach? Eur J Hosp Pharm Sci Pract 2015;22:243-8.

34 Walsh KA, O'Riordan D, Kearney PM, et al. Improving the appropriateness of prescribing in older patients: a systematic review and meta-analysis of pharmacists' interventions in secondary care. Age Ageing 2016;45:201-9.
35 Cullinan S, O'Mahony D, Byrne S. Application of the structured history taking of medication use tool to optimise prescribing for older patients and reduce adverse events. Int J Clin Pharm 2016;38:374-9.

36 Lee DH, Vielemeyer 0 . Analysis of overall level of evidence behind Infectious Diseases Society of America practice guidelines. Arch Intern Med 2011;171:18-22.

37 Tricoci P, Allen JM, Kramer JM, et al. Scientific evidence underlying the ACC/AHA clinical practice guidelines. JAMA 2009;301:831-41.

38 Fried TR, Tinetti ME, lannone L. Primary care clinicians' experiences with treatment decision-making for older persons with multiple conditions. Arch Intern Med 2011;171:75-80.

39 Damestoy N, Collin J, Lalande R. Prescribing psychotropic medication for elderly patients: some physicians' perspectives. CMAJ 1999;161:143-5.

40 Dickinson $\mathrm{R}$, Knapp $\mathrm{P}$, House $\mathrm{AO}$, et al. Long-term prescribing of antidepressants in the older population: a qualitative study. Br J Gen Pract 2010;60:e144-55.

41 Gordon M. Non-technical skills training to enhance patient safety. Clin Teach 2013; 10:170-5

42 Beers MH, Ouslander JG, Rollingher I, et al. Explicit criteria for determining inappropriate medication use in nursing home residents. UCLA Division of Geriatric Medicine. Arch Intern Med 1991;151:1825-32. 